\title{
Biomaterials
}

\section{Enhanced cell survival of melanocyte spheroids in serum starvation condition}

\author{
Sung-Jan Lin ${ }^{\mathrm{a}, \mathrm{b}, \mathrm{c}, \mathrm{d}}$, Shiou-Hwa Jee ${ }^{\mathrm{b}, \mathrm{d}}$, Wen-Chu Hsiao ${ }^{\mathrm{a}}$, Hsin-Su Yu ${ }^{\mathrm{b}, \mathrm{d}}$, Tsen-Fang Tsai ${ }^{\mathrm{b}, \mathrm{d}}$, \\ Jau-Shiuh Chen ${ }^{\text {b,d }}$, Chih-Jung Hsu ${ }^{\text {b,d }}$, Tai-Horng Young ${ }^{a, *}$ \\ ${ }^{a}$ Institute of Biomedical Engineering, College of Medicine and College of Engineering, National Taiwan University, \#1, \\ Section 1, Jen-Ai Road, Taipei 100, Taiwan \\ ${ }^{\mathrm{b}}$ Department of Dermatology, National Taiwan University Hospital, \#7, Chung-Shan South Road, Taipei 100, Taiwan \\ ${ }^{\mathrm{c}}$ Department of Dermatology, Yun-Lin Branch of National Taiwan University Hospital, Douliu, Yunlin, Taiwan \\ ${ }^{\mathrm{d}}$ Department of Dermatology, National Taiwan University College of Medicine, \#1, Section 1, Jen-Ai Road, Taipei 100, Taiwan
}

Received 19 April 2005; accepted 23 August 2005

Available online 19 September 2005

\begin{abstract}
Autologous melanocyte transplantation for vitiligo treatment by use of melanocyte suspension has drawbacks including cell damage in cell preparation and transportation, difficult manipulation and low engraftment rate in acral vitiligious lesions. We have proposed the concept of cellular patch as an alternative solution. In the development of melanocyte patches, we have shown that chitosan membrane supports the growth and phenotype expression of melanocytes. Surprisingly, melanocytes spontaneously grow into three-dimensional spheroids on chitosan-coated surface. In this work, we demonstrate that, compared with monolayered melanocytes, melanocyte spheroids show a better survival in growth factor and serum-deprived condition. Survival of melanocytes is further ameliorated when a greater portion of melanocytes is precultured into spheroidal morphology. Melanocyte spheroids disintegrate and the cells return to a physiological dendritic morphology after they are reinoculated on collagen I-coated surface. Our results show that melanocytes are morphologically transformable depending on the substratum used and spheroidal melanocytes have a superior survival to that of monolayered dendritic melanocytes in stringent conditions. Preculturing melanocytes into spheroids can provide melanocytes a survival advantage. Chitosan-based melanocyte patch can be a promising method to enhance the engraftment rate and facilitate the cell preparation and transplantation procedures in melanocyte transplantation for vitiligo treatment.
\end{abstract}

(C) 2005 Elsevier Ltd. All rights reserved.

Keywords: Melanocytes; Spheroids; Chitosan; Vitiligo; Cellular patch; Cell survival

\section{Introduction}

Vitiligo, a common acquired depigmented disease is characterized by destruction of functional melanocytes in the epidermis [1,2]. Non-invasive treatment for vitiligo includes topical steroid, psoralen plus UVA (PUVA), narrow-band UVB, and low energy laser irradiation [1-3]. These treatments are aimed at reactivating dormant melanocytes, presumably located in the hair follicle, to

\footnotetext{
*Corresponding author. Tel.: + $886223123456 \times 1455$; fax: +886223940049 .

E-mail address: thyoung@ha.mc.ntu.edu.tw (T.-H. Young).
}

repopulate the depigmented epidermis [1,4-6]. In difficult cases, autologous melanocyte transplantation using cultured melanocytes has been used [2,7-10]. In the preparation of cells for transplantation, the cells are trypsinized into suspension. The cell suspension is then applied on the dermabraded depigmented lesions during transplantation. However, difficulty in handling the melanocyte suspension and variable successful repigmentation rates is often encountered. For example, it takes efforts to evenly spread melanocytes on the lesions during transplantation. When the lesional skin is rugged, seeding and secure attachment of melanocytes onto the skin is very challenging. Further, the success rates for repigmentation for lesions on acral 
parts and periorificial areas are very disappointing $[2,10]$. Correction of depigmentation on these areas is of invaluable cosmetic, psychological and social significance.

To overcome drawbacks using melanocyte suspension, the concept of using tissue-engineered "cellular patch" for melanocyte transplantation has been proposed in our previous publication [11]. The cellular patch is made by preculturing melanocytes on a biomaterial membrane and the biomaterial-melanocyte patch can be used to cover the lesional sites with an upside-down orientation during transplantation. It can simplify the process in preparing cells for transplantation and also facilitate the transplantation procedures. In the development of melanocyte patch, we have shown that chitosan is a good candidate for use as the membranous part of the patch [11]. It supports the growth and phenotype expression of human melanocytes [11]. Similar to neuron cells, human melanocytes adopt a dendritic morphology both in vivo and in conventional culture condition [12]. Surprisingly, human melanocytes formed three-dimensional multicellular spheroids on chitosan-coated surface when an appropriate seeding density was used [11]. Up to this point, the biological function of the melanocyte spheroid was unknown.

In this work, we demonstrate that human melanocytes have a better survival in growth factor and serum deprived condition when they are precultured into multicellular spheroids on chitosan-coated surface. The enhanced survival is further ameliorated when a greater portion of melanocytes is cultured into spheroidal morphology before growth factor and serum are deprived. Further, spheroidal melanocytes grow back into physiological dendritic melanocytes when they are re-inoculated on collagen I-coated surface. Hence, chitosan-based melanocyte patch can be a promising method to enhance the engraftment rate and facilitate the cell preparation and transplantation procedures in autologous melanocyte transplantation for vitiligo treatment.

\section{Materials and methods}

\subsection{Preparation of culture wells coated with chitosan}

A $15 \mathrm{mg} / \mathrm{ml}(\mathrm{W} / \mathrm{V})$ solution of chitosan (C-3646, Sigma, USA, $\mathrm{Mn}=810,000 \mathrm{gm} / \mathrm{mole}$, degree of deacetylation $=85 \%$ ) was prepared by dissolving chitosan in $1 \mathrm{~m}$ acetic acid. For preparing chitosan-coated wells, $0.5 \mathrm{ml}$ of chitosan solution was added into each well of 24-welled tissue culture polystyrene plates (Costar, USA). The solution was then allowed to dry at $50^{\circ} \mathrm{C}$ for 2 days to form a thin membrane. Each well was then neutralized by $0.1 \mathrm{~N} \mathrm{NaOH}$ aqueous solution for $15 \mathrm{~min}$ and washed thoroughly with double-distilled water. Before cell culture, the prepared chitosan-coated wells were sterilized in $70 \%$ alcohol overnight and rinsed extensively with phosphate buffered saline (PBS), followed by treatment under ultraviolet light overnight. As controls, uncoated polystyrene wells were treated by the same way as chitosan-coated wells.

\subsection{Cell culture, cell death assays and cell survival assays}

Human melanocytes were provided by cell bank of Department of Dermatology, National Taiwan University Hospital. The study protocol was approved by our Institutional Review Board. We conformed to the Helsinki
Declaration with respect to human subjects in biomedical research. Human melanocytes are cultured using modified melanocyte medium (MMM) as previously described [11]. Passage 1 or 2 melanocytes are detached by trypsinization before seeding. Cells were seeded evenly in the wells at the density of $25 \times 10^{3} / \mathrm{cm}^{2}$ in the following experiments unless stated otherwise.

To investigate the survival of melanocytes in serum starvation condition, the cells were first cultured in the chitosan-coated wells using MMM. In control groups, uncoated tissue culture polystyrene wells were used. The culture medium was aspirated and the cells were rinsed twice with PBS before the medium was changed to starvation medium DMEM (DMEM, BIOSOURCE INC., USA) at indicated times described in the result.

It has been shown that growth factor withdrawal induces death of human melanocytes [13]. Qualitative and quantitative determination of melanocyte death during growth factor and serum deprivation was performed using 7Aminoactinomycin D (7-AAD) staining and lactate dehydrogenase efflux assay at indicated times $[14,15]$. For 7-AAD staining, cells in the supernatant were collected by centrifugation and cells attached to the culture wells were obtained by trypsinization. The total cells collected from the same well were mixed and rinsed with PBS twice and then incubated in 7-AAD (Sigma, USA) solution ( $1 \mathrm{mg} / \mathrm{ml}$ in PBS) for $20 \mathrm{~min}$ at $4{ }^{\circ} \mathrm{C}$ in the dark. The cells were collected by centrifugation and then rinsed thoroughly with PBS twice. Then the cells were transferred to a precoated glass slide. The cells were inspected and photographed with a Nikon fluorescence microscopy. For determination of lactate dehydrogenase released by dead cells into the medium, lactate dehydrogenase efflux assay using the supernatant was performed by use of a cytotoxicity detection kit at indicated times according to the product manual (Cytotoxicity Detection Kit, Cat. No. 1644793, Roche, Germany). The lactate dehydrogenase efflux was determined by the optical density at $490 \mathrm{~nm}$ obtained from an enzyme-linked immunosorbent assay (ELISA) plate reader (ELx800, BIOTEK).

Quantitative analysis of cell survival was determined by MTT assays at the indicated times [11,16], because counting of cells in the spheroids involves vigorous mechanical dissociation which damages the cells and results in a lower accuracy than automated quantification. The 300- $\mu$ l MTT (Sigma, USA) solution $(2 \mathrm{mg} / \mathrm{ml}$ in PBS) was added to each well. After $3 \mathrm{~h}$ incubation at $37^{\circ} \mathrm{C}$, the supernatant was discarded and dimethyl sulfoxide (Merck, Germany) of $200 \mu \mathrm{l}$ was added to dissolve the formazan crystals. The dissolvable solution was jogged homogenously for $15 \mathrm{~min}$ at room temperature by the shaker. The solution of each well was transferred to an eppendorf and was centrifuged at 1500 r.p.m. for $1 \mathrm{~min}$ to remove any possible melanocytes contaminated in the solution. The optical density of $100 \mu \mathrm{l}$ of the formazan solution was read on an ELISA plate reader (ELx800, BIOTEK) at $570 \mathrm{~nm}$. For morphological observation, the cells are also photographed by digital camera coupled to a phase contrast microscopy.

\subsection{Behavior of melanocyte spheroids on collagen-coated surface}

Because transplanted melanocytes make direct contact with dermabraded dermis which is composed mainly of collagen I, we investigated the behavior of melanocyte spheroids on the collagen I-coated surface. Glass coverslips were coated with collagen I as previously described [17]. After cultured on chitosan-coated wells for 5 days when melanocytes formed compact spheroids, the melanocyte spheroids were removed from chitosan-coated wells by use of a pipette carefully without disturbing the spheroids. The spheroids were transferred to a tissue culture well containing a coverslip pre-coated with collagen I and then cultured in MMM. The morphology of the cells were photographed under phase contrast microscope at indicated times.

\section{Results}

\subsection{Survival advantage of melanocyte spheroids in growth factor and serum deprived condition}

When melanocytes were cultured on chitosancoated wells, they formed multicellular spheroids on day 
3 (Figs. 1(a), (b)) and larger and more compact spheroids were observed on day 5 (Figs. 1(c), 1(d)). Compared with cells on day 3 , monolayered dendritic melanocytes distributed between the spheroids decreased on day 5. On the contrary, no spheroid formation was observed in the melanocytes cultured on tissue culture polystyrene plate after incubation for 5 days (Fig. 1(e)).

The cells precultured in MMM on chitosan-coated wells for 3 days (Chi3) and 5 days (Chi5), respectively, were used in determination of survival upon growth factor and serum deprivation. In the control group, cells precultured in MMM on tissue culture polystyrene plate for 3 days (TCP3) and 5 days (TCP5) were used. Qualitative determination of melanocyte death was performed using 7-AAD staining on day 5. Quantitative determination of melanocyte death was performed using lactate dehydrogenase efflux test on day 5, day 7 and day 9 after serum starvation. Baseline data of the two tests were obtained from each group just before the culture medium was changed to starvation medium. In 7-AAD staining, increased dead cells were seen in all 4 groups on day 5 as compared with baseline data. The representative pictures of Chi5 cells after serum starvation for 5 days are shown in Fig. 2. Dead cells are permeable to 7-AAD and their nuclei are stained red under fluorescence microscopy (Fig. 2(b)). The result of lactate dehydrogenase efflux test was shown in Fig. 3 as optical density. In Fig. 3(a), significantly lower lactate dehydrogenase efflux was observed in melanocytes precultured on chitosan-coated wells for 3 days (Chi3) as compared with the cells precultured on tissue culture polystyrene plate for 3 days (TCP3) at all indicated times. It indicates that less cell death was induced in melanocytes precultured on chitosan-coated surface for 3 days. A similar result was observed in melanocytes precultured for
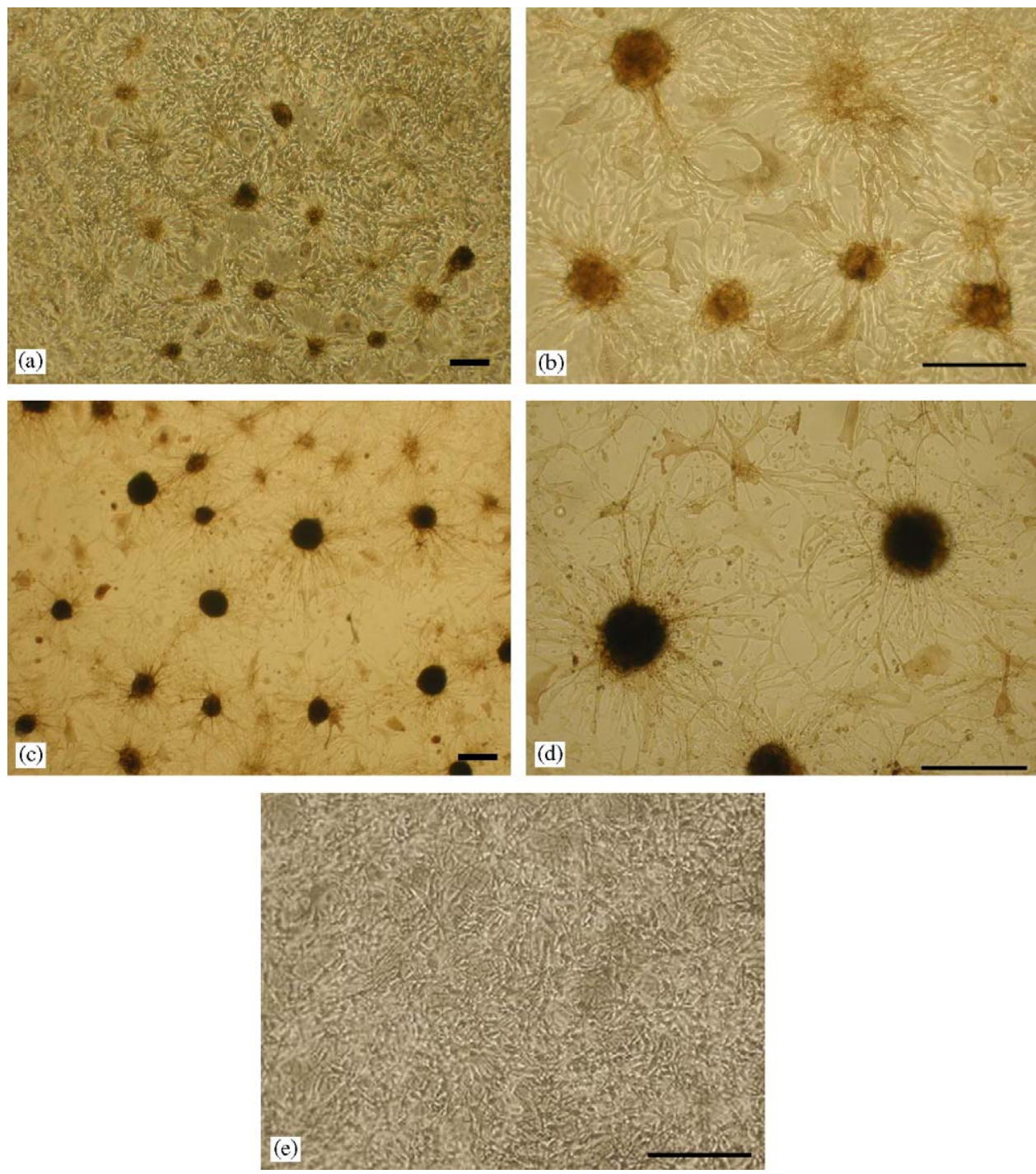

Fig. 1. Morphology of melanocytes precultured on chitosan-coated wells and tissue culture polystyrene wells. (a, b) Melanocyte spheroids can be observed on chotisan-coated wells on day 3. (c, d) Larger and more compact melanocyte spheroids form on chotisan-coated wells on day 5. (e) Melanocytes remain monolayred on tissue culture polystyrene wells on day 5. (Phase contrast, bars $200 \mu \mathrm{m}$.) 

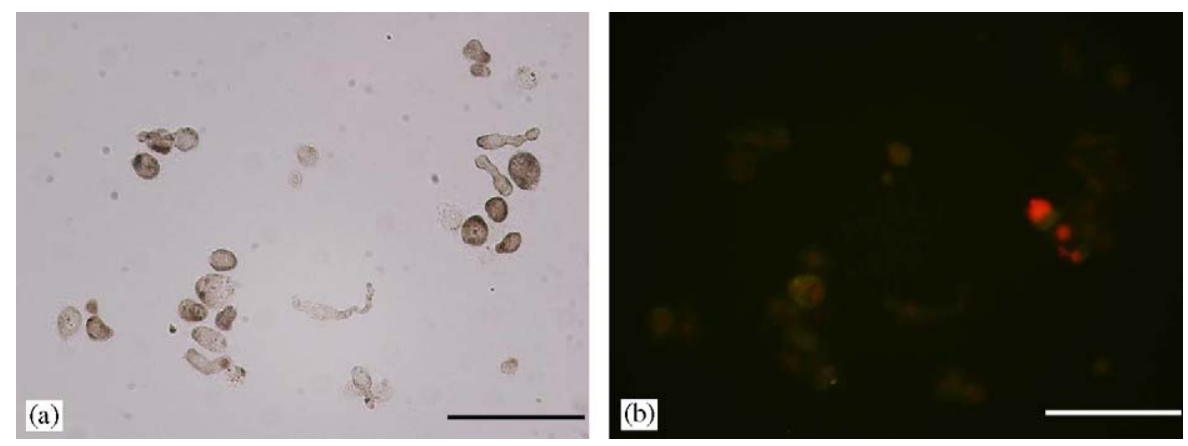

Fig. 2. Light and fluorescence micrographs of 7-AAD stained Chi5 melanocytes after 5 days in serum deprived condition. (a) Light micrograph of Chi5 cells. (b) The same field under fluorescence microscopy and nuclei of dead cells are stained red. (Bars $50 \mu \mathrm{m})$.
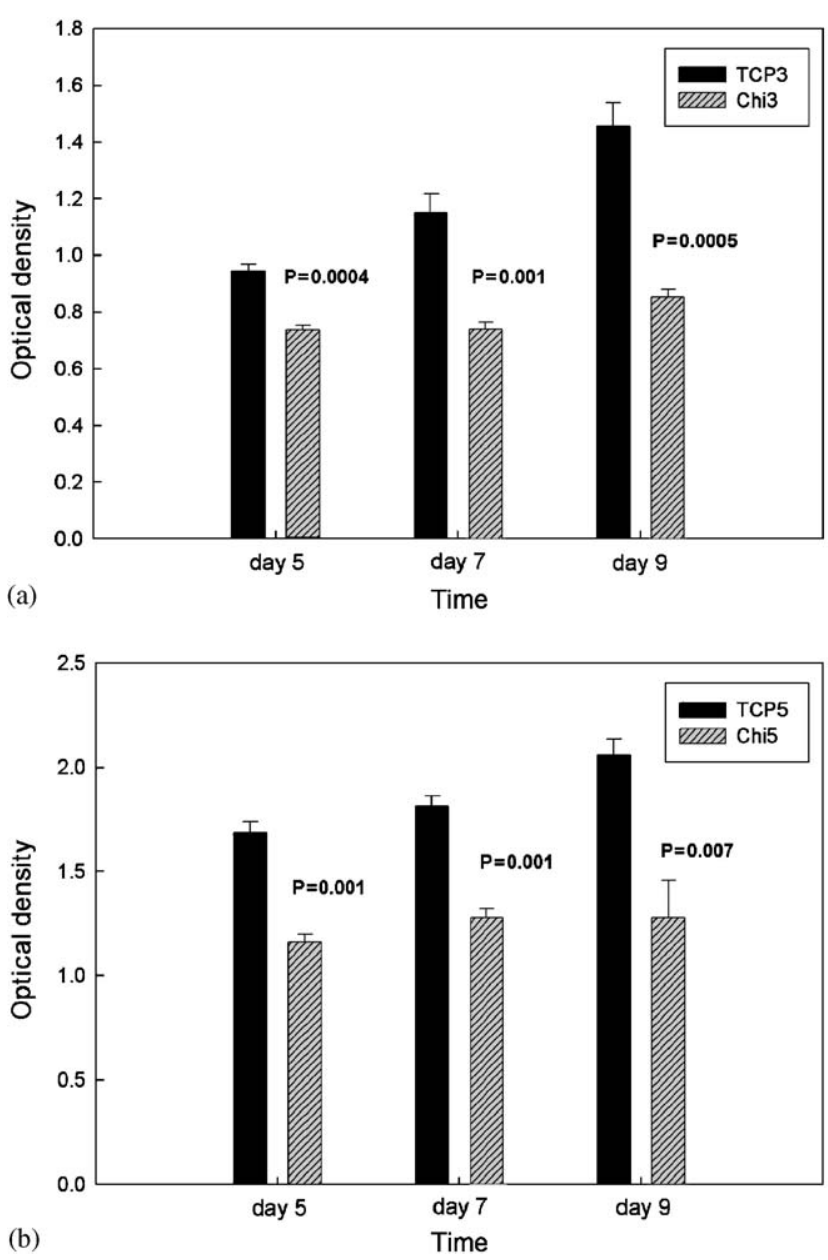

Fig. 3. Lactate dehydrogenase efflux assay of melanocytes after serum starvation for 5, 7 and 9 days. (a) At all indicated times during serum starvation, less lactate dehydrogenase efflux was observed in cells precultured on chitosan-coated wells for 3 days (Chi3) as compared with that precultured on tissue culture polystyrene wells for 3 days (TCP3). (b) Similarly, less lactate dehydrogenase efflux was revealed in cells precultured on chitosan-coated wells for 5 days (Chi5) as compared with that precultured on tissue culture polystyrene wells for 5 days (TCP5) at all indicated times. Error bars reflect SEM from three or four independent experiments and cells were derived from the same donor foreskin in all experiments. Student's $t$ test was performed for comparison and each $\mathrm{p}$ value is shown in the figure.

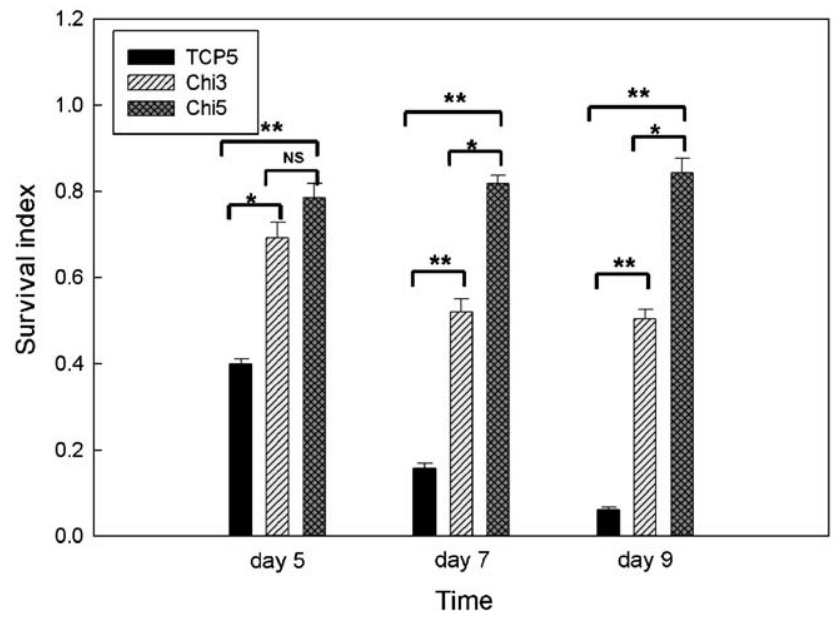

Fig. 4. Survival indices of melanocytes after serum starvation for 5, 7 and 9 days. (TCP5: melanocytes precultured on tissue culture polystyrene wells for 5 days before serum starvation; Chi3: melanocytes precultured on chitosan-coated wells for 3 days before serum starvation; Chi5: melanocytes precultured on chitosan-coated wells for 5 days before serum starvation). Error bars reflect SEM from three or four independent experiments and cells were derived from the same donor foreskin in all experiments. Student's $t$ test was performed for comparison as indicated. $\left({ }^{*} p<0.001 ;{ }^{* *} p<0.0001 ;\right.$ NS: non-significant $)$.

5 days on chitosan-coated wells (Chi5) and tissue culture polystyrene plate (TCP5) (Fig. 3(b)). Hence, monolayered melanocytes precultured on tissue culture polystyrene plate are more liable to death induced by growth factor and serum deprivation as compared with cells precultured on chitosan-coated surface.

Quantitative analysis of cell survival was performed using MTT tests 5, 7, and 9 days after the medium was changed to starvation medium. MTT test was also performed in each group just before the medium was changed to starvation medium as baseline value. A survival index is defined as the proportion of absorbance value at $570 \mathrm{~nm}$ at each indicated time to the baseline absorbance value at $570 \mathrm{~nm}$ of each group. The result is shown in Fig. 4. Quantitative comparison of survival indices of TCP5 and Chi3 on day 5, day 7 and day 9 
after growth factor and serum deprivation reveals that the survival of Chi3 cells is superior to that of TCP5 cells at all indicated times. The survival index of TCP5 continues to decrease as long as the cells are kept in erum starvation condition, indicating a deteriorating trend of cell survival in the TCP5 group with time. On the contrary, the survival index of Chi3 only slightly decrease from day 5 to day 7 and remains almost unchanged from day 7 to day 9 .

In addition, it is noted that the survival index of Chi5 is significantly greater than that of Chi3 from day 7 to day 9 (Fig. 4). On the contrary to that of Chi3, survival index of Chi5 does not decrease from day 5 to day 7 . Instead, survival index of Chi5 slightly increases from day 5 to day 9 , suggesting that, even in serum-deprived condition, Chi5 cells not only can survive but also have the ability to grow. Overall, in comparison with monolayered dendritic melanocytes, spheroidal morphology render melanocytes advantageous in survival and the larger the spheroid, the more significant the advantage. Hence, melanocytes precultured on chitosan-coated surface for a longer period have a more superior survival advantage in serum starvation condition.

\subsection{Spheroidal melanocytes remain an aggregated morphology in growth factor and serum deprived condition}

The morphology of Chi3 cells before and during serum starvation is shown in Fig. 5. Morphology of TCP5 cells is also shown for comparison. Compared with the morphology of melanocytes before serum deprivation (Fig. 5(a)), monolayered cells between the spheroids are greatly reduced after starvation for 1 day (Fig. 5(b)). It is possible that serum starvation may accelerate the transformation of monolayered melanocytes into spheroids on chitosancoated surface. However, it may be that cells in a monolayered morphology on chitosan-coated surface are more vulnerable to serum deprivation and detach from chitosan-coated surface when they are no longer viable after starvation for 1 day. Hence, a larger proportion of cells in monolayered morphology may attenuate the overall survival of Chi3 cells as compared with that of Chi5 cells. From day 1 to day 5, melanocyte spheroids became larger (Figs. 5(b)-(d)), presumably due to mergence of melanocyte spheroids formed on day 1 . On the contrary, cells of TCP5 remained monolayered during the serum deprivation period for 3 days (Fig. 5(f)) and cells progressively
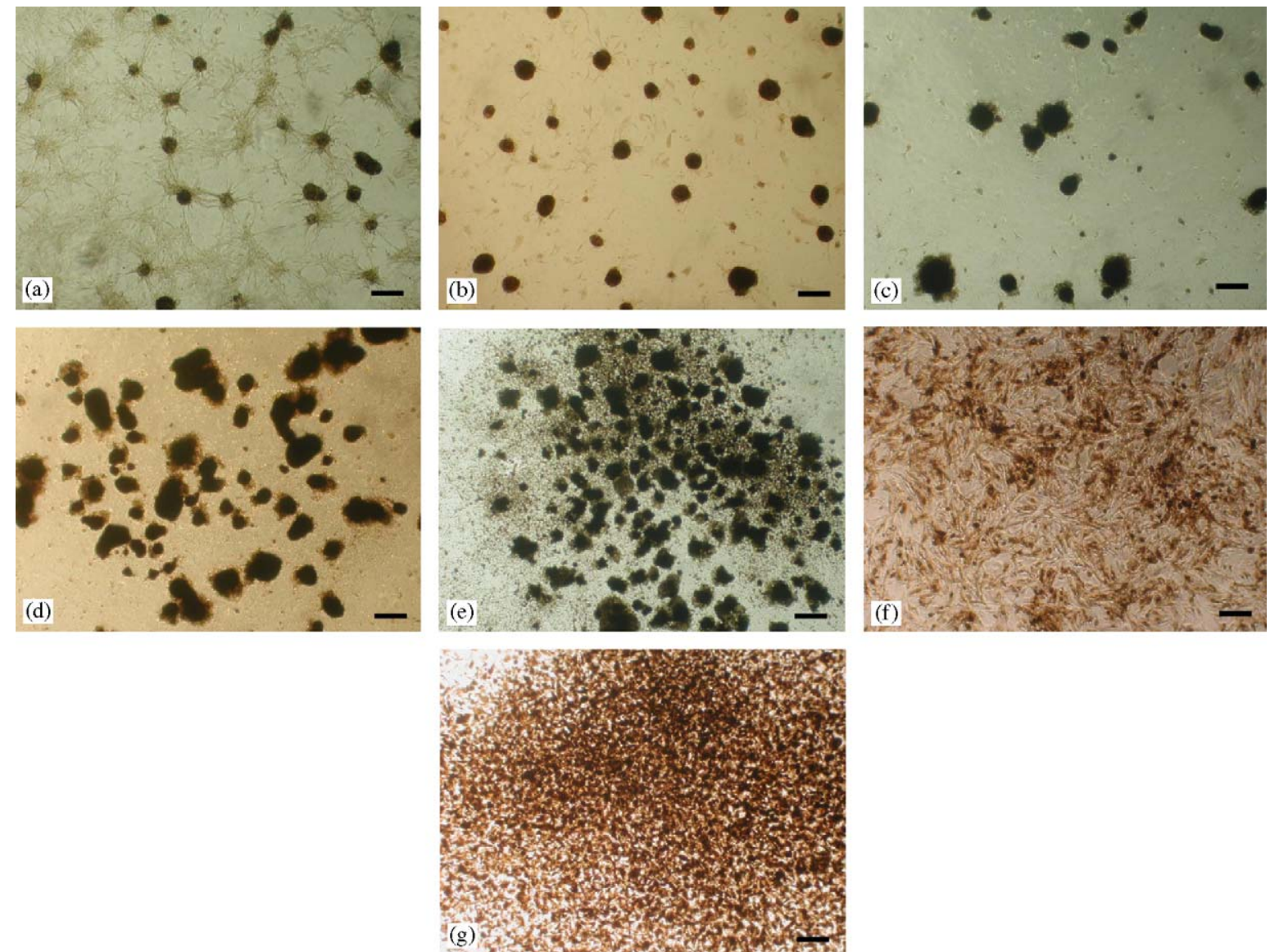

Fig. 5. Cell morphology of Chi3 and TCP5 cells in serum-deprived condition. (a) Chi3 cells before serum deprivation. (b) Chi3 cells 1 day after serum deprivation. (c) Chi3 cells 3 days after serum deprivation. (d) Chi3 cells 5 days after serum deprivation. (e) Chi3 cells 7 days after serum deprivation. (f) TCP5 cells 3 days after serum deprivation. (g) TCP5 cells 7 days after serum deprivation. (Phase contrast, bars $200 \mu \mathrm{m}$.) 
detached from the culture plates as they stayed longer in serum deprivation (Fig. 5(g)). This corresponds to the decreasing trend of survival of TCP5 cells in Fig. 4. The spheroids become partially disintegrated on the periphery on day 7 (Fig. 5(e)), suggesting that cells at the spheroidal surface might lose viability from day 5 to day 7 . This conclusion was compatible with the result in Fig. 4 in which the survival index of Chi3 decreased from day 5 to day 7 .

\subsection{Melanocyte spheroids are capable of growing into monolayered dendritic melanocytes when they are reinoculated on collagen I-coated surface}

The morphology of melanocyte spheroids reinoculated on collagen I-coated surface is shown in Fig. 6. After reinoculated on collagen-coated surface, monolayered dendritic melanocytes gradually grew out from the spheroids and the spheroids gradually disintegrated. This further demonstrates that spheroidal melanocytes are viable and morphologically transformable depending on the substratum used. This is of physiological significance in terms of melanocyte transplantation. Since collagen $\mathrm{I}$ is the major component of dermis, melanocyte spheroids will directly contact collagen I in the exposed dermis of dermabraded depigmented skin in transplantation. Hence, spheroidal melanocytes will grow back to its physiological dendritic morphology after being in contact with dermis.

\section{Discussion}

Melanocytes, derived from neural crest, migrate to skin in early embryonic stage [18-20]. Usually, melanocytes are not able to secrete growth factors by themselves $[1,12]$. The function and survival of melanocytes in the epidermis depends on supporting signals and trophic factors from the surrounding cells and environment [12,21-29]. Keratinocytes play a major role in regulating the function and growth of melanocytes by direct contact and via secretion of growth factors including basic fibroblast growth factor (bFGF), nerve growth factor, endothelin 1 [21-23].
Cultured melanocytes are also highly dependent on external stimuli for growth and survival $[1,12]$.

Low engraftment efficiency remains a main problem in treating acral and periorificial vitiliginous lesions using melanocyte suspension transplantation $[2,10]$. Though the detailed process of initial engraftment of transplanted melanocytes to the recipient sites is not fully characterized, it is suggested that acral skin, compared with skin of other part of the body, is less capable of maintaining transplanted melanocytes [2]. There are several possible factors affecting the survival of transplanted cells using melanocyte suspension. First, melanocytes might be damaged during the cell suspension preparation procedures. Typsinization and mechanical agitation to suspend the cells can injure melanocytes before transplantation. Second, the condition of melanocytes will deteriorate when they are kept in suspension. A variety of cells undergo apoptosis (anoikis) when they are kept in suspension [30,31]. Anoikis can be a problem in melanocyte transplantations when cell suspension is used. This point has been emphasized in other transplantations using cell suspension. For example, in cell preparation for hepatocyte transplantation, it has been shown that loss of anchorage triggers apoptosis in primary hepatocytes within $15 \mathrm{~min}$ [32]. During melanocyte transplantation, it takes time for the cell and skin preparation before melanocyte suspension is applied on the dermabraded skin. The initial attachment of melanocytes to the recipient area cannot be ensured using cell suspension. Therefore, melanocyte survival will be curtailed by loss of anchorage for such a long time. Third, the growth condition in the recipient area is stringent for melanocytes. In preparation of melanocyte suspension, the growth factors and trophic factors are removed from the medium. The dermabraded area is initially deficient in keratinocytes that play a major role as a supporter for melanocyte survival in physiological conditions. Without exogenous trophic factors and survival signals from keratinocytes, the condition is very stern for melanocyte growth.

How to provide a "survival advantage" is critical in promoting the rates of successful engraftment. It has been
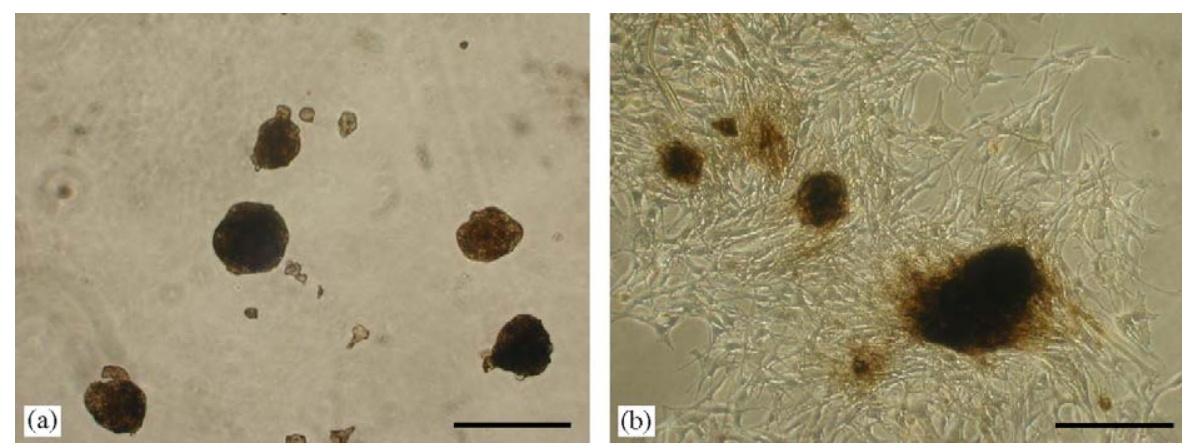

Fig. 6. Changes of cell morphologies of melanocyte spheroids on collagen I-coated surface. (a) Morphology of melanocyte spheroids immediately after they were reinoculated on collagen I-coated surface. (b) Melanocytes spheroids gradually disintegrated and grew into monolayered dendritic cells 3 days after reinoculated on collagen I-coated surface. (Phase contrast, bars $200 \mu \mathrm{m}$.) 
shown that better healing and tissue regeneration can be achieved by adding exogenous growth factors [33-36]. However, extremely high doses of growth factors should be provided owing to rapid clearance and degradation of growth factors in vivo, making this method uneconomical. In melanocyte transplantation, we can also be confronted by difficulties in determining the optimal concentration of growth factors and maintaining therapeutic concentration in vivo. Another method to yield survival advantage can be theoretically achieved by enhancing the expression of growth factors by the transplanted cells themselves. However, this is potentially hazardous in the case of melanocytes because it has been shown that genetically engineered melanocytes overexpressing growth factors can be transformed to a phenotype similar to melanoma [37].

It has been reported that death is gradually induced in human melanocytes when trophic factors are withdrawn (but in the presence of serum factors) [13]. To simulate the stringent conditions melanocytes may encounter during transplantation, both trophic factors and serum are deprived in our experiment to investigate the survival behavior of melanocyte spheroids. Our results show that melanocytes precultured on chitosan-coated surface have a superior survival to that of monolayered melanocytes in serum starvation. This enhanced survival is associated with the morphological transformation from monolayered dendritic cells into multicellular spheroids, indicating that the survival advantage of transplanted melancoytes can be achieved by preculturing melanocytes on chitosan-coated surface without genetic manipulation and the addition of trophic factors into the transplantation sites. This is of great potential in clinical application for its convenience and economy.

We also demonstrate that melanocytes have two interchangeable morphologies according to the substratum used. Spheroidal morphology can enhance the survival of melanocytes during transplantation and melanocytes can spontaneously change back to a dendritic morphology similar to that in physiological condition after contacting collagen I, the major extracellular matrix of dermis. As we suggested, the morphology of melanocytes may depend on two competing forces: cell-cell interaction and cell-substratum interaction [11]. Therefore, the interaction between collagen I and melanocytes should be stronger than that between chitosan and melanocytes.

The mechanism for superior survival of melanocyte spheroids is currently unknown. We have previously shown that increased cell density enhanced the survival of neurons in stringent conditions via autocrine or paracrine secretion of growth factors [38]. It has also been shown that intimate intercellular contact in cell clusters can enhance the growth factor secretion in cancer cell lines [39]. We are currently analyzing the possible role of secreted growth factors by melanocyte spheroids themselves in the enhanced survival.

In addition to cell survival, a number of difficulties in manipulation of cell suspension during melanocyte transplantation remain to be solved. Cell suspension is incon- venient for use. It is difficult to uniformly spread melanocytes on the recipient site. It takes time when the lesion is extensive or very rugged. Another challenge is the control of concentration of cell suspension. With higher content of fluid, the suspension tends to flow out of the dermabraded area or accumulate on focal dented areas of the recipient site. On the contrary, when the fluid content in the cell suspension is diminished, it is difficult to uniformly spread cells on the recipient site; the suspension will easily dry out and compromise the survival of transplanted cells. Furthermore, during melanocyte transplantation, the constant contact and attachment of melanocytes to the recipient skin cannot be ensured using cell suspension. This point is more obvious in acral and periorificial lesions where the skin is constantly motile and may contribute in part to the disappointingly low engraftment rates in these areas. These drawbacks will not be encountered by use of melanocyte patch. The transplantation procedures can be facilitated because the transplantation can be easily completed by applying the patch to the dermabraded area. The attachment of transplanted cells to the dermabraded area is more secured in this way. In highly motile sites, the patch can be anchored to the skin by use of appropriate dressings, bandages or even sutures.

Using chitosan-based melanocyte patch for melancoyte transplantation has other advantages as well. No trypsinization is needed during the cell preparation before transplantation. It not only simplifies the cell preparation but also reduces possible damage to the cells by trypsinization and loss of anchorage. Insulation from the environment by chitosan membranes provides transplanted cells a more favorable growth condition. In addition, chitosan can enhance the skin wound healing and reduce the recovery period for the patient [40-43]. The antimicrobial property of chitosan may reduce the possible infection during transplantation [44]. Its transparency also makes it easier to monitor the cell behavior and possible contamination during culture period and after transplantation.

\section{Conclusion}

We have succeeded in providing melanocytes survival advantage in stringent conditions by preculturing melanocytes into melancoyte spheroids on chitosan-coated surface. This method is convenient and very economical since trysinization procedures and exogenous growth factors are not needed. Chitosan-based melanocyte patch can be a promising method to facilitate the transplantation procedures and improve engraftment rates in melanocyte transplantation.

\section{References}

[1] $\mathrm{Yu}$ HS. Melanocyte destruction and repigmentation in vitiligo: a model for nerve cell damage and regrowth. $J$ Biomed Sci 2002;9:564-73. 
[2] Njoo MD, Westerhof W, Bos JD, Bossuyt PMM. The development of guidelines for treatment of vitiligo. Arch Dermatol 1999; 135:1514-21.

[3] Yu HS, Wu CS, Yu CL, Kao YH, Chiou MH. Helium-neon laser irradiation stimulates migration and proliferation in melanocytes and induces repigmentation in segmental-type vitiligo. J Invest Dermatol 2003;120:56-64.

[4] Staricco RG. Activation of amelanotic melanocytes in the outer root sheath of the hair follicle following ultraviolet exposure. J Invest Dermatol 1962;39:163-4.

[5] Ortonne JP, Schmitt D, Thivolet J. PUVA-induced repigmentation of vitiligo: scanning electron microscopy of hair follicles. J Invest Dermatol 1980;74:40-2.

[6] Cui J, Shen LY, Wang GC. Role of hair follicles in the repigmentation of vitiligo. J Invest Dermatol 1991;97:410-6.

[7] Lontz W, Olsson MJ, Moellmann G, Lerner AB. Pigment cell transplantation for treatment of vitiligo: a progress report. J Am Acad Dermatol 1994;30:591-7.

[8] Chen YF, Yang PY, Hung CM, Huang MH, Hu DN. Autotransplantation in segmental vitiligo by using cultured pure melanocytes: analysis of 19 treated cases. Dermatol Sin 1999;17:193-9.

[9] Guerra L, Capurro S, Melchi F, Primavera G, Bondanza S, Cancedda R, et al. Treatment of "stable" vitiligo by timedsurgery and transplantation of cultured epidermal autografts. Arch Dermatol 2000;136:1380-9.

[10] Yarr M. Vitiligo: the evolution of cultured epidermal autografts and other surgical treatment modalities. Arch Dermatol 2001;137: 348-9.

[11] Lin SJ, Jee SH, Shiao WC, Lee SJ, Young TH. Formation of melanocyte spheroids on the chitosan-coated surface. Biomaterials 2005;26:1413-22.

[12] Yaar M, Gilchrest BA. Human melanocyte growth and differentiation: a decade of new data. J Invest Dermatol 1991;97:611-7.

[13] Alanko T, Rosenburg M, Saksela O. FGF expression allows nevus cells to survive in three-dimensional collagen gel under conditions that induce apoptosis in normal human melanocytes. J Invest Dermatol 1999;113:111-6.

[14] Gaforio JJ, Serrano MJ, Algarra I, Ortega E, Alvarez de Cienfuegos G. Phagocytosis of apoptotic cells assessed by flow cytometry using 7Aminoactinomycin D. Cytometry 2002;49:8-11.

[15] Koh JY, Choi DW. Quantitative determination of glutamate mediated cortical neuronal injury in cell culture by lactate dehydrogenase efflux assay. J Neurosci Method 1987;20:83-90.

[16] Mosmann T. Rapid colorimetric assay for cellular growth and survival: application of proliferation and cytotoxicity assays. J Immunol Methods 1983;65:55-63.

[17] Beck AJ, Phillips J, Smith-Thomas L, Short RD, MacNeil S. Development of a plasma-polymerized surface suitable for the transplantation of keratinocyte-melanocyte cocultures for patients with vitiligo. Tissue Eng 2003;9:1123-31.

[18] Rawles ME. Origin of pigment cells from neural crest in the mouse embryo. Physiol Zool 1947;20:248-66.

[19] Boissy RE, Nordlund JJ. Molecular basis of congenital hypopigmentary disorders in humans: a review. Pigm Cell Res 1997;10:12-24.

[20] Dupin E, Le Douarin NM. Development of melanocyte precursors from the vertebrate neural crest. Oncogene 2003;22:3016-23.

[21] Halaban R, Langdon R, Birchall N, Cuono C, Baird A, Scott G, et al. Basic fibroblast growth factor from human keratinocytes is a natural mitogen for melanocytes. J Cell Biol 1988;107:1611-9.

[22] Yaar M, Grossman K, Eller M, Gilchrest BA. Evidence for nerve growth factor-mediated paracrine effects in human epidermis. J Cell Biol 1991;115:821-8.

[23] Imokawa G, Yada Y, Miyagosh M. Endothelins secreted from human keratinocytes are intrinsic mitogens for human melanocytes. J Biol Chem 1992;267:24675-80.

[24] Yaar M, Eller MS, DiBenedetto P, Reenstra WR, Zhai S, McQuaid $\mathrm{T}$, et al. The trk family of receptors mediates nerve growth factor and neurotrophin-3 effects in melanocytes. J Invest Dermatol 1994;94:1550-62.

[25] Hara M, Yaar M, Gilchrest BA. Endothelin-1 of keratinocyte origin is a mediator of melanocyte dendricity. $\mathbf{J}$ Invest Dermatol 1995; 105:744-8.

[26] Nakazawa K, Nakazawa H, Collombel C, Damour O. Keratinocyte extracellular matrix-mediated regulation of normal human melanocyte function. Pigm Cell Res 1995;8:10-8.

[27] Romero-Graillet C, Aberdam E, Clement M, Ortonne JP, Ballotti R. Nitric oxide produced by ultraviolet-irradiated keratinocytes stimulates melanogenesis. J Clin Invest 1997;99:635-42.

[28] Kippenberger S, Bernd A, Bereiter-Hahn J, Ramirez-Bosca A, Kaufmann R. The mechanism of melanocyte dendrite formation: the impact of differentiating keratinocytes. Pigm Cell Res 1998;11:34-7.

[29] Moellmann G, Halaban R. Growth factor receptors and signal transduction regulating the proliferation and differentiation of melanocytes. In: Nordlund JJ, Boissy RE, Hearing VJ, King RA, editors. The pigmentary system: physiology and pathophysiology. Oxford: Oxford University Press; 1998. p. 135-49.

[30] Frisch SM, Francis H. Disruption of epithelial cell-matrix interactions induces apoptosis. J Cell Biol 1994;124:619-26.

[31] Grossmann J, Walther K, Artinger M, Kiessling S, Scholmerich J. Apoptotic signaling during initiation of detachment-induced apoptosis ("anoikis") of primary human intestinal epithelial cells. Cell Growth Differ 2001;12:147-55.

[32] Smets FN, Chen Y, Wang LJ, Soriano HE. Loss of cell anchorage triggers apoptosis (anoikis) in primary mouse hepatocytes. Mol Genet Metab 2002;75:344-52.

[33] Canalis E, McCarthy TL, Centrella M. Effects of platelet-derived growth factor on bone formation in vitro. J Cell Physiol 1989;140:530-7.

[34] Lynch SE, Castella GR, Williams RC, Kiritsy CP, Howell TH, Reddy MS, et al. The effects of short-term application of a combination of platelet-derived and insulin-like growth factors on periodontal wound healing. J Periodontol 1991;62:458-67.

[35] Rutherford RB, Niekrash CE, Kennedy JE, Charette MF. Plateletderived and insulin-like growth factors stimulate regeneration of periodontal attachment in monkeys. J Periodont Res 1992;27:285-90.

[36] Park JB, Matsuura M, Han KY, Norderyd O, Lin WL, Genco RJ, et al. Periodontal regeneration in class III furcation defects of beagle dogs using guided tissue regenerative therapy with platelet-derived growth factor. J Periodontol 1995;66:462-77.

[37] Nesbit M, Nesbit HKE, Bennett J, Andl T, Hsu MY, Dejesus E, et al. Basic fibbroblast growth factor induces a transformed phenotype in normal human melanocytes. Oncogene 1999;18:6469-76.

[38] Young TH, Huang JH, Huang SH, Hsu JP. The role of cell density in the survival of cultured cerebellar granule neurons. J Biomed Mater Res 2000;52:748-53.

[39] Laderoute KR, Murphy BJ, Short SM, Grant TD, Knapp AM, Sutherland RM. Enhancement of transforming growth factor-a synthesis in multicellular tumour spheroids of A431 squamous carcinoma cells. Br J Cancer 1992;65:157-62.

[40] Muzzarelli RA, Mattioli-Belmonte M, Pugnaloni A, Biagini G. Biochemistry, histology and clinical uses of chitins and chitosans in wound healing. EXS 1999;87:251-64.

[41] Cho YW, Cho YN, Chung SH, Yoo G, Ko SW. Water-soluble chitin as a wound healing accelerator. Biomaterials 1999;20:2139-45.

[42] Stone CA, Wright H, Clarke T, Powell R, Devaraj VS. Healing at skin graft donor sites dressed with chitosan. Br J Plast Surg 2000;53:601-6.

[43] Ishihara M, Nakanishi K, Ono K, Sato M, Kikuchi M, Saito Y, et al. Photocrosslinkable chitosan as a dressing for wound occlusion and accelerator in healing process. Biomaterials 2002;23:833-40.

[44] Rabea EI, Badawy ME, Stevens CV, Smagghe G, Steurbaut W. Chitosan as antimicrobial agent: applications and mode of action. Biomacromolecules 2003;4:1457-65. 